EESTI NSV TEADUSTE AKADEEMIA TOIMETISED, XII KÖIDE FOOSIKA-MATEMAATIKA- JA TEHNIKATEADUSTE SEERIA, 1963, NR. 4

ИЗВЕСТИЯ АКАДЕМИИ НАУК ЭСТОНСКОИ ССР. ТОМ ХІІ СЕРИЯ ФИЗИКО-МАТЕМАТИЧЕСКИХ И ТЕХНИЧЕСКИХ НАУК. 1963, № 4

\title{
К ТЕОРИИ НЕСИММЕТРИЧНЫХ ЗЕРКАЛ
}

\section{П. КАРД,}

\author{
член-корреспондент Академии наук Эстонской ССР
}

\section{Введение}

Основы теории интерференшионных несимметричных зеркал (отражателей) были изложены в опубликованной ранее статье [1]. Там были найдены условия, необходимые и достаточные для того, чтобы полупрозрачное зеркало, имеющее заданный коэффнщиент пропускания $D$, было максимально несимметричным, т е., чтобы его коэффициент отражения $R$ при падении света, с одной стороны, был равен нулю, а с другой стороны, был возможно большим. В этом смысле изложенная в указанной статье теория является исчерпываюшей. Однако форма, в которой получены условия несимметричности, не является единственно возможной. Часто бывает удобно пользоваться другими условиями несимметричности, по существу эквивалентными прежним, но сильно отличающимися от них по форме. Знание тех и других условий представляется в целях их практического использования весьма существенным, так как позволяет выбирать наиболее удобную форму в каждой конкретной задаче, в зависимости от ее характера.

Цель настоящей статьи и заключается в выводе новой формы условий несимметричности. Тем самым теория принимает законченный вид. Кроме того, хотя эквивалентность старых и новых условий будет ясна из общих соображений, мы проведем также необходимые выкладки, чтобы показать их взаимную связь непосредственно.

\section{Постановка задачи}

Многослойное несимметричное зеркало должно содержать по крайней мере один поглощающий слой. Поэтому его целесообразно рассматривать состоящим из трех частей, разделенных промежуточными непоглощающими слоями (последние можно считать присутствующими в пленке даже тогда, когда их на самом деле нет, так как им можно приписать нулевую толщину). Промежуточные слои выбираются так. чтобы крайние части пленки были тоже непоглощающими, т. е. в среднюю часть должны войти все поглощающие слои пленки. Обозначим ограничивающие среды индексами 0 и 3 , а промежуточные слои индексами 1 и 2. Схема пленки показана на рис. 1. Величины, относящиеся ко всей пленке, будем обозначать индексами 03 или 30 (в зависимости от направления падения света), а величины, относящиеся к отдельным частям - соответственно другими парами индексов (например, 01,31 и т. д.).

Дадим сводку основных обозначений и соотно шений, которымн мы будем пользоваться.

Амплитудные коэффициенты отражения и пропускания всей пленки или какой-либо ее части обозначим через $r$ и $d$, а энергетические коэффициентм, через $R$ и $D$, так что

0

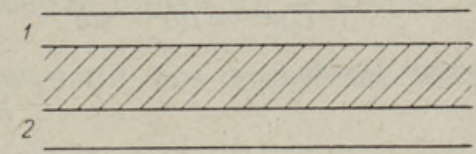

Рис. 1. 


$$
\left.\begin{array}{l}
R=r r^{*} \\
D=d d^{*}
\end{array}\right\}
$$

(* означает комплексное сопряжение). А будет обозначать коэффициент поглощения, так что

$$
R+D+A=1 \text {. }
$$

Знак - означает у нас пленку, сопряженную с данной (о понятии сопряжения см. в другой статье $\left.\left[{ }^{2}\right]\right)$. Величины $a, b, \mu, v$ определяются равенствами:

$$
\left.\begin{array}{l}
a=\frac{r}{d}=\sqrt{\frac{R}{D}} e^{i \mu} \\
b=\frac{1}{d}=\frac{1}{\sqrt{D}} e^{i^{\nu}} .
\end{array}\right\}
$$

Отдельные слои характеризуются величиной

$$
\alpha=k n h \cos \theta,
$$

где $k$ - волновое число в вакууме, $n$ и $h$ - показатель преломления и толщина слоя. $\theta-$ угол преломления в нем. Для любой пленки имеют место формулы

$$
b \tilde{b}^{*}-a \tilde{a}^{*}=1
$$

и

$$
\left.\begin{array}{l}
a_{k i}=-\tilde{a}_{i k}^{*} \\
b_{k i}=b_{i k}
\end{array}\right\}
$$

( $i$ и $k$ - индексы ограничивающих пленку сред). Если пленка состоит из двух частей, разделенных промежуточным слоем" (индекс $m$ ), то

$$
\left.\begin{array}{l}
a_{i k}=a_{i m} b_{m k} e^{i \alpha_{m}}+a_{m k} \tilde{b}_{i m} e^{-i \alpha_{m}} \\
b_{i k}=b_{i m} b_{m k} e^{i \alpha_{m}}+a_{m k} \tilde{a}_{i m}^{*} e^{-i \alpha_{m}} .
\end{array}\right\}
$$

Наконец, вещественные величины $S$ и $\varphi$ и комплексная величина Ueia определяются так:

$$
\begin{aligned}
\operatorname{ch} \varphi & =1+\frac{1}{2}\left(\frac{A}{D}+\frac{\tilde{A}}{\tilde{D}}\right)= \\
& =\frac{1}{2}\left(b b^{*}-a a^{*}+\tilde{b} \tilde{b}^{*}-\tilde{a} \tilde{a}^{*}\right) \\
S & =\frac{1}{2}\left(\frac{A}{D}-\frac{\tilde{A}}{\tilde{D}}\right)=\frac{1}{2}\left(b b^{*}-a a^{*}-\tilde{b} \tilde{b}^{*}+\tilde{a} \tilde{a}^{*}\right) \\
U e^{i \sigma} & =\tilde{a} b-a \tilde{b}
\end{aligned}
$$

причем всегда

$$
S^{2}-U^{2}=\operatorname{sh}^{2} \varphi
$$

Величина $\varphi$ всякой пленки определяется только ее поглощающей частью и не зависит от направления падения света. Поэтому мы будем писать ф без индексов (т. е. $\varphi_{03}=$ $\left.=\varphi_{30}=\varphi_{12}=\varphi_{21} \equiv \varphi\right)$. У непоглощающей пленки $\varphi$ равно нулю.

Для того чтобы пленка 03 была несимметричным зеркалом, нужно, чтобы коэффициент отражения, с одной стороны (например, сверху на рис. 1), был равен нулю, а с другой стороны, был возможно велик. В следующем разделе рассмотрим условия, необходимые и достаточные для этого. 


\section{Условия несимметричности}

В упомянутой статье [ $\left.{ }^{1}\right]$ показано (в несколько иных обозначениях), что для $R_{03}=0$ необходимо и достаточно, чтобы

$$
\begin{gathered}
R_{23}=\tilde{R}_{20} \\
\cos \left(2 \alpha_{2}+\mu_{32}+v_{32}-\tilde{\mu}_{20}+\tilde{v}_{20}\right)=-1
\end{gathered}
$$

Этот результат получается, если рассматривать пленку 03 состоящей из пленок 02 и 23, и применить формулы (6) и (7). Кроме того, там показано, что в случае выполнения этих условий все коэффициенты отражения, пропускания и поглощения выражаются так:

$$
\left.\begin{array}{l}
R_{03}=0 \\
D_{03}=\operatorname{ch} \varphi-S_{20} \\
A_{03}=1+S_{20}-\operatorname{ch} \varphi ; \\
R_{30}=U_{20}^{2}=S_{20}^{2}-\operatorname{sh}^{2} \varphi \\
D_{30}=\operatorname{ch} \varphi-S_{20} \\
A_{30}=\left(\operatorname{ch} \varphi-S_{20}\right)\left(\operatorname{ch} \varphi+S_{20}-1\right) .
\end{array}\right\}
$$

Отсюда вытекает, что при заданном $\varphi$ эти величины зависят еще от $S_{20}$. Эта величина, в свою очередь, зависит от состава пленки так:

$$
S_{20}=\frac{1+R_{01}}{1-R_{01}} S_{21}+2 U_{21} \frac{\sqrt{R_{01}}}{1-R_{01}} \cos \left(2 \alpha_{1}+\sigma_{21}-\mu_{10}+v_{10}\right) .
$$

Теперь рассмотрим другой способ вывода условий несимметричности, приводящий к иным по форме результатам. Для этого примем пленку 03 состоящей из пленок 01 и 13. Прежде всего, согласно первой формуле (7) имеем

$$
\frac{R_{0}}{D_{03}}=\frac{1}{D_{01} D_{13}}\left(R_{01}+R_{13}+2 \sqrt{R_{01} R_{13}} \cos \left(2 \alpha_{1}+\mu_{01}+v_{01}-\mu_{13}+v_{13}\right)\right) .
$$

Отсюда следует, что для $R_{03}=0$ необходимо и достаточно, чтобы выполнялись следующие условия:

$$
R_{01}=R_{13}
$$

и

$$
\cos \left(2 \alpha_{1}+\mu_{01}+v_{01}-\mu_{13}+v_{13}\right)=-1 \text {. }
$$

Первое условие требует определенного выбора пленки 01, а второе надлежащего выбора толщины слоя 1.

Вычислим, полагая эти условия выполненными, все остальные коэффициенты. По второй формуле (7) имеем

$$
\frac{1}{D_{03}}=\frac{1}{D_{01} D_{13}}\left(1+R_{01} R_{13}+2 \sqrt{R_{01} R_{13}} \cos \left(2 \alpha_{1}+\mu_{01}+v_{01}-\mu_{13}+v_{13}\right)\right) \text {. }
$$

Подставляя сюда $R_{13}$ вместо $R_{01}$ и -1 вместо косинуса, получим

$$
\frac{1}{D_{03}}=\frac{\left(1-R_{13}\right)^{2}}{\left(1-R_{13}\right) D_{13}}=\frac{A_{13}+D_{13}}{D_{13}}=\operatorname{ch} \varphi+S_{13} .
$$


Таким образом,

$$
D_{03}=\frac{1}{\operatorname{ch} \varphi+S_{13}}
$$

Вычислим также $R_{30}$. Согласно (6) и (7)

$$
a_{30}=-\tilde{a}_{13}^{*} b_{01} e^{i \alpha_{1}}-a_{01}^{*} \tilde{b}_{13}^{*} e^{-i \alpha_{1}},
$$

откуда следует

$$
\frac{R_{30}}{D_{30}}=\frac{1}{D_{01} \tilde{D}_{13}}\left(R_{01}+\tilde{R}_{13}+2 \sqrt{R_{01} \tilde{R}_{13}} \cos \left(2 \alpha_{1}+\mu_{01}+v_{01}-\tilde{\mu}_{13}+\tilde{v}_{13}\right)\right) .
$$

Учитывая (15) и (16), отсюда имеем

$$
\begin{gathered}
\frac{R_{s 0}}{D_{30}}=\frac{D_{13}}{1-R_{13}}\left(\frac{R_{13}}{D_{13} \tilde{D}_{13}}+\frac{\tilde{R}_{13}}{D_{13} \tilde{D}_{13}}-\frac{2 \sqrt{R_{13} \tilde{R}_{13}}}{D_{13} \tilde{D}_{13}} \cos \left(\mu_{13}-v_{13}-\right.\right. \\
\left.\left.-\tilde{\mu}_{13}+\tilde{v}_{13}\right)\right)=\frac{D_{13}}{A_{13}+D_{13}}\left(\tilde{a}_{13} b_{13}-a_{13} \tilde{b}_{13}\right)\left(\tilde{a}_{13}^{*} b_{13}-a_{13} \tilde{b}_{13}^{*}\right)= \\
=\frac{U_{13}^{2}}{\operatorname{ch} \varphi+S_{13}} .
\end{gathered}
$$

А так как

$$
D_{30}=D_{03}=\frac{1}{\operatorname{ch} \varphi+S_{13}}
$$

To

$$
R_{30}=\frac{U_{13}^{2}}{\left(\operatorname{ch} \varphi+S_{13}\right)^{2}} .
$$

Коэффициенты поглощения $A_{03}$ и $A_{30}$ находятся согласно (2). Полный результат таков:

$$
\left.\begin{array}{l}
R_{03}=0 \\
D_{03}=\frac{1}{\operatorname{ch} \varphi+S_{13}} \\
A_{03}=\frac{\operatorname{ch} \varphi+S_{13}-1}{\operatorname{ch} \varphi+S_{13}} ;
\end{array}\right\}
$$

Эти формулы аналогичны формулам (12) и (13). Подобно тем, в них, кроме $\varphi$, входит еще $S_{13}$. Эта величина, аналогично $S_{20}$ в (14), выражается так:

$$
S_{13}=\frac{1+R_{23}}{1-R_{23}} S_{12}+2 U_{12} \frac{\sqrt{R_{23}}}{1-R_{23}} \cos \left(2 \alpha_{2}+\sigma_{12}-\mu_{23}+v_{23}\right) .
$$


В некоторых случаях важно знать, кроме коэффициентов $R, D$ и $\boldsymbol{A}$, также те же величины для сопряженной пленки. $\tilde{D}_{03}$ можно вычислить аналогично тому, как сделано выше для $D_{03}$. Из второй формулы (7) следует

$$
\frac{1}{\tilde{D}_{03}}=\frac{1}{D_{01} \tilde{D}_{13}}\left(1+R_{01} \tilde{R}_{13}+2 \sqrt{R_{01} \tilde{R}_{13}} \cos \left(2 \alpha_{1}+\mu_{01}+v_{01}-\tilde{\mu}_{13}+\tilde{v}_{13}\right)\right) .
$$

Учитывая (15) и (16), имеем далее

$$
\frac{1}{\tilde{D}_{03}}=\frac{D_{13}}{A_{13}+D_{13}}\left(b_{13} \tilde{b}_{13}^{*}-a_{13} \tilde{a}_{13}\right)\left(b_{13}^{*} \tilde{b}_{13}-a_{13}^{*} \tilde{a}_{13}\right)=\frac{1}{\operatorname{ch} \varphi+S_{13}} .
$$

Итак,

$$
\tilde{D}_{03}=\operatorname{ch} \varphi+S_{13}
$$

Остальные коэффициенты можно найти уже весьма просто. Из первой формулы (6) следует, что

$$
\frac{\tilde{R}_{03}}{\tilde{D}_{03}}=\frac{R_{30}}{D_{30}}=\frac{U_{13}^{2}}{\operatorname{ch} \varphi+S_{13}} \quad \text { и } \quad \frac{\tilde{R}_{30}}{\tilde{D}_{30}}=\frac{R_{03}}{D_{03}}=0 .
$$

$\tilde{A}_{03}$ и $\tilde{A}_{30}$ находим по формуле (2). Окончательно имеем

$$
\begin{aligned}
& \tilde{D}_{03}=\tilde{D}_{30}=\operatorname{ch} \varphi+S_{13} \\
& \tilde{R}_{03}=U_{13}^{2}=S_{13}^{2}-\operatorname{sh}^{2} \varphi \\
& \tilde{R}_{30}=0 \\
& \tilde{A}_{03}=\left(\operatorname{ch} \varphi+S_{13}\right)\left(\operatorname{ch} \varphi-S_{13}-1\right) \\
& \tilde{A}_{30}=1-\operatorname{ch} \varphi-S_{13} .
\end{aligned}
$$

\section{Эквивалентность двух вариантов условий несимметричности}

Условия (10) и (11), с одной стороны, и (15) и (16), с другой стороны, кажутся на первый взгляд не только не эквивалентными, но даже противоречащими друг другу. В самом деле, условие (11) заставляет выбрать определенным образом толщину только второго промежуточного слоя, тогда как толщина первого остается произвольной и определяет только $S_{20}$ (согласно (14)). По условию (16) и равенству (21) дело обстоит как раз наоборот: толщина первого промежуточного слоя фиксируется, а второго остается произвольной. Однако это противоречие является кажущимся, так как в условия (11) и (16) входят также величины $\mu_{13}$, $v_{13}, \tilde{\mu}_{20}$ и $\tilde{v}_{20}$. Поэтому толщины обоих промежуточных слоев на самом деле соопределяются условиями несимметричности. Толщину одного из них можно выбирать произвольно, и тогда толщина другого определяется условием несимметричности, но притом именно так, что если мы эту толщину выберем как произвольную, то толщина первого слоя определится такой, какой она была принята произвольно сначала. То же относится и к коэффициентам отражения крайних частей 01 и 23, соопределяемых условиями (10) и (15). Эквивалентность тех и других усло- 
вий вытекает просто из того, что и те и другие являются необходимыми и достаточными для $R_{03}=0$. Представляется нелишним, однако, убедиться в этом и с помощью прямого вычисления. Итак, покажем, каким образом из условий (10) и (11) вытекают равенства (15) и (16).

Вычислим сначала $R_{13}$. Исходя из формул $(3)$ и $(7)$ и учитывая $(10)$ и (11), имеем

$$
\begin{gathered}
R_{13}=\frac{R_{13} / D_{13}}{1 / D_{13}}=\frac{\left|a_{12} b_{23} e^{i \alpha_{2}}+a_{23} \tilde{b}_{12}^{*} e^{-i \alpha_{2}}\right|^{2}}{\mid b_{12} b_{23} e^{i \alpha_{2}+\left.a_{23} \tilde{a}_{12}^{*} e^{-i \alpha_{2}}\right|^{2}}=} \\
=\frac{\frac{1}{D_{23}}\left(\frac{R_{12}}{D_{12}}+\frac{R_{23}}{\tilde{D}_{12}}+2 \sqrt{\frac{R_{12} R_{23}}{D_{12} \tilde{D}_{12}}} \cos \left(2 \alpha_{2}+\mu_{12}+\tilde{v}_{12}-\mu_{23}+v_{23}\right)\right)}{\frac{1}{D_{23}}\left(\frac{1}{D_{12}}+\frac{R_{23} \tilde{R}_{12}}{\tilde{D}_{12}}+2 \sqrt{\frac{R_{23} \tilde{R}_{12}}{D_{12} \tilde{D}_{12}}} \cos \left(2 \alpha_{2}+\tilde{\mu}_{12}+v_{12}-\mu_{23}+v_{23}\right)\right)}= \\
=\frac{\frac{1}{\tilde{D}_{20}}\left(\frac{R_{12}}{D_{12}}+\frac{\tilde{R}_{20}}{\tilde{D}_{12}}-2 \sqrt{\frac{R_{12} \tilde{R}_{20}}{D_{12} \tilde{D}_{12}}} \cos \left(\mu_{12}+\tilde{v}_{12}+\tilde{\mu}_{20}-\tilde{v}_{20}-\mu_{23}-\mu_{32}\right)\right)}{\tilde{D}_{20}\left(\frac{1}{D_{12}}+\frac{\tilde{R}_{20} \tilde{R}_{12}}{\tilde{D}_{12}}-2 \sqrt{\frac{\tilde{R}_{12} \tilde{R}_{20}}{D_{12} \tilde{D}_{12}}} \cos \left(\tilde{\mu}_{12}+v_{12}+\tilde{\mu}_{20}-\tilde{v}_{20}-\mu_{23}-\mu_{32}\right)\right)} .
\end{gathered} .
$$

Но, согласно (6), $\mu_{23}+\mu_{32}=\pi$. Следовательно,

$$
R_{13}=\frac{\left|a_{12}^{*} \tilde{b}_{20}+\tilde{a}_{20} \tilde{b}_{12}\right|^{2}}{\left|b_{12}^{*} \tilde{b}_{20}+\tilde{a}_{20} \tilde{a}_{12}\right|^{2}} .
$$

Подставляя сюда, согласно (7),

$$
\left.\begin{array}{l}
\tilde{a}_{20}=-a_{12}^{*} b_{01} e^{i \alpha_{1}}-a_{01}^{*} b_{12}^{*} e^{-i \alpha_{1}} \\
\tilde{b}_{20}=b_{01} \tilde{b}_{12} e^{i \alpha_{1}}+\tilde{a}_{12} a_{01}^{*} e^{-i \alpha_{1}}
\end{array}\right\}
$$

и учитывая формулу (5), находим

$$
R_{13}=\frac{a_{01} a_{01}^{*}}{b_{01} b_{01}^{*}}=R_{01},
$$

что и совпадает с (15).

Во-вторых, найдем

$$
\cos \left(2 \alpha_{1}+\mu_{01}+v_{01}-\mu_{13}+v_{13}\right) .
$$

Для этого напишем

$$
\begin{gathered}
\frac{\sqrt{R_{13}}}{D_{13}} e^{i\left(-\mu_{13}+v_{13}\right)}=a_{13}^{*} b_{13}= \\
=\left(a_{12}^{*} b_{23}^{*} e^{-i a_{2}}+a_{23}^{*} \tilde{b}_{12} e^{i a_{2}}\right)\left(b_{12} b_{23} e^{i a_{2}}+a_{23} \tilde{a}_{12}^{*} e^{-i a_{2}}\right)= \\
=a_{12}^{*} b_{12} b_{23} b_{23}^{*}+\tilde{a}_{12}^{*} \tilde{b}_{12} a_{23} a_{23}^{*}+ \\
+a_{23}^{*} b_{23} b_{12} \tilde{b}_{12} e^{2 i a_{2}}+a_{23} b_{23}^{*} a_{12}^{*} \tilde{a}_{12}^{*} e^{-2 i a_{2}}
\end{gathered}
$$


Согласно (11) отсюда следует

$$
\begin{aligned}
& \frac{\sqrt{R_{13}}}{D_{13}} e^{i\left(-\mu_{13}+v_{13}\right)}=\frac{1}{D_{23}}\left[a_{12}^{*} b_{12}+R_{23} \tilde{a}_{12}^{*} \tilde{b}_{12}+\right. \\
&\left.+\sqrt{R_{23}} b_{12} \tilde{b}_{12} e^{i\left(\tilde{\mu}_{20}-\tilde{v}_{20}\right)}+\sqrt{R_{23}} a_{12}^{*} \tilde{a}_{12}^{*} e^{-i\left(\tilde{1}_{20}-\tilde{v}_{90}\right)}\right] .
\end{aligned}
$$

Подставляя сюда $\tilde{R}_{20}$ вместо $R_{23}$, имеем

$$
\begin{gathered}
\frac{D_{23} \sqrt{R_{13}}}{\tilde{D}_{20} D_{13}} e^{i\left(-\mu_{13}+v_{13}\right)}= \\
=a_{12}^{*} b_{12} \tilde{b}_{20} \tilde{b}_{20}^{*}+\tilde{a}_{12}^{*} \tilde{b}_{12} \tilde{a}_{20} \tilde{a}_{20}^{*}+\tilde{a}_{20} \tilde{b}_{20}^{*} b_{12} \tilde{b}_{12}+\tilde{a}_{20}^{*} \tilde{b}_{20} a_{12}^{*} \tilde{a}_{12}^{*}= \\
=\left(a_{12}^{*} \tilde{b}_{20}+\tilde{a}_{20} \tilde{b}_{12}\right)\left(b_{12} \tilde{b}_{20}^{*}+\tilde{a}_{20}^{*} \tilde{a}_{12}^{*}\right) .
\end{gathered}
$$

Подставляя теперь сюда вместо $\tilde{a}_{20}$ и $\tilde{b}_{20}$ выражения из формул (23), находим

$$
\frac{D_{23} V \overline{R_{13}}}{\tilde{D}_{20} D_{13}} e^{i\left(-\mu_{13}+v_{13}\right)}=-a_{01}^{*} b_{01}^{*} e^{-2 i \alpha_{1}}
$$

Отсюда с учетом (15) имеем

$$
\frac{D_{01} D_{23}}{D_{13} \tilde{D}_{20}} e^{i\left(2 \alpha_{1}+\mu_{01}+v_{01}-\mu_{13}+v_{13}\right)}=-1 .
$$

Отсюда вытекает (16), что и требовалось показать.

Вдобавок равенство (24) дает еще

$$
\frac{D_{01} D_{23}}{D_{13} \tilde{D}_{20}}=1,
$$

или, учитывая, что $D_{23}=1-R_{23}=1-\tilde{R}_{20}=\tilde{D}_{20}+\tilde{A}_{20}$

и $D_{01}=1-R_{01}=1-R_{13}=D_{13}+A_{13}$,

$$
\left(1+\frac{A_{13}}{D_{13}}\right)\left(1+\frac{\tilde{A}_{20}}{\tilde{D}_{20}}\right)=1,
$$

T. e.

$$
\left(\operatorname{ch} \varphi+S_{13}\right)\left(\operatorname{ch} \varphi-S_{20}\right)=1 .
$$

Это равенство может быть получено и другим путем из формул (14), (15) и (16). В самом деле, из этих формул вытекает:

$$
\begin{gathered}
\text { chr }-S_{20}=\operatorname{ch} \varphi-\frac{1+R_{13}}{1-R_{13}} S_{21}-2 U_{21} \frac{\sqrt{R_{13}}}{1-R_{13}} \cos \left(\sigma_{21}+\mu_{13}-v_{13}\right)= \\
=\frac{\operatorname{ch} \varphi-S_{21}-R_{13}\left(\operatorname{ch} \varphi+S_{21}\right)-2 U_{21} \sqrt{R_{13}} \cos \left(\sigma_{21}+\mu_{13}-v_{13}\right)}{A_{13}+D_{13}}= \\
=\frac{\frac{1}{D_{13}}\left(1+\frac{\tilde{A}_{21}}{\tilde{D}_{21}}\right)-\frac{R_{13}}{D_{13}}\left(1+\frac{A_{21}}{D_{21}}\right)-\frac{2 U_{21} \sqrt{R_{13}}}{D_{13}} \cos \left(\sigma_{21}+\mu_{13}-v_{13}\right)}{1+\frac{A_{13}}{D_{13}}}=
\end{gathered}
$$




$$
\begin{aligned}
& =\frac{b_{13} b_{13}\left(\tilde{b}_{21} \tilde{b}_{21}^{*}-\tilde{a}_{21} \tilde{a}_{21}^{*}\right)-a_{13} a_{13}\left(b_{21} b_{21}^{*}-a_{21} a_{21}\right)-}{\operatorname{ch} \varphi+S_{13}}+ \\
& +\frac{-a_{13} b_{13}^{*}\left(\tilde{a}_{21} b_{21}-a_{21} \tilde{b}_{21}\right)-a_{13}^{*} b_{13}\left(\tilde{a}_{21}^{*} b_{21}^{*}-a_{21}^{*} \tilde{b}_{21}^{*}\right)}{\operatorname{ch} \varphi+S_{13}} \text {. }
\end{aligned}
$$

Очевидно, чтобы получить отсюда (25), нужно, чтобы числитель этого выражения был равен единице. В самом деле, раскрывая скобки и перегруппировывая члены, приходим к выражению

$$
\begin{aligned}
& \left(b_{13}^{*} \tilde{b}_{12}-a_{13}^{*} \tilde{a}_{12}\right)\left(b_{13} \tilde{b}_{12}^{*}-a_{13} \tilde{a}_{12}^{*}\right)- \\
& -\left(b_{13} a_{12}-a_{13} b_{12}\right)\left(b_{13}^{*} a_{12}^{*}-a_{13}^{*} b_{12}^{*}\right) .
\end{aligned}
$$

Если подставим сюда вместо $a_{13}$ и $b_{13}$ их выражения по формулам (7), то получим $b_{23} b_{23}-a_{23} a_{23}$, что по формуле (5) действительно равно единице.

Из формулы (24) вместе с формулами (12) - (13) и (19) - (20) вытекает, что оба варианта условий несимметричности дают одинаковые значения всех коэффициентов $R, D$ и $A$. Это и естественно, в силу эквивалентности обоих вариантов. Удобно выразить, исключая $S_{13}$ или $S_{20}$, все коэффициенты $R, A, \tilde{D}, \tilde{R}$ и $\tilde{A}$ через ч и коэффициент пропускания. Опуская индексы у $D$, запишем результаты в виде

$$
\begin{aligned}
& R_{03}=0 \\
& A_{03}=1-D \\
& R_{30}=1-2 D \operatorname{ch} \varphi+D^{2} \\
& A_{30}=D(2 \operatorname{ch} \varphi-1-D)
\end{aligned}
$$

H

$$
\begin{aligned}
& \tilde{D}_{03}=\tilde{D}_{30}=\frac{1}{D} \\
& \tilde{R}_{03}=\frac{1-2 D \operatorname{ch} \varphi+D^{2}}{D^{2}} \\
& \tilde{A}_{03}=\frac{D(2 \operatorname{ch} \varphi-1)-1}{D^{2}} \\
& \tilde{R}_{30}=0 \\
& \tilde{A}_{30}=\frac{D-1}{D} .
\end{aligned}
$$

\section{Обсуждение и графическое представление результатов}

На рис. 2 показаны графики $D$ и $R_{30}$ в зависимости от $D$ и $\varphi$, вычисленные по формулам -(26). Диагональ квадрата есть график $D$ в зависимости от $D$, причем значения $D$ отсчитываются по ординате снизу. Кривые линии (параболы) являются графиками $R_{30}$ в зависимости от $D$, а $\varphi$ является параметром. Значения $R_{30}$ отсчитываются по ординате сверху. Таким образом, проведенная в любом месте вертикальная прямая линия делится внутри квадрата диагональю и какой-либо параболой на три части, изоборажающие значения $D_{30}, A_{30}$ и $R_{30}$ (снизу вверх). Из графиков видно, что при фиксированном значении $\varphi$ несимметрич- 


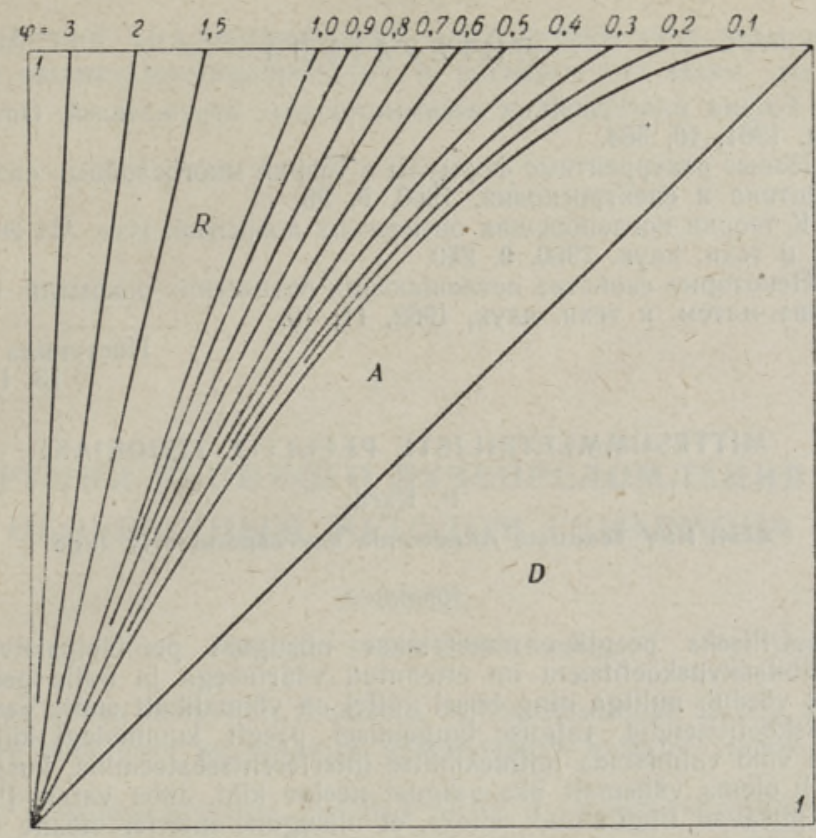

Pнс. 2.

ность тем больше, чем меньше $D$. Вообще, $D$ не может быть больше $e^{-\varphi}$, и при $D=e^{-\varphi}, R_{30}=0$, т. е. несимметричность исчезает. На графике значениям $D=e^{-\varphi}$ соответствуют точки пересечения парабол с верхней горизонтальной стороной квадрата. Из чертежа видно также, что при выбранном значении $D$ несимметричность тем больше, чем меньше $\varphi$ (это видно также из формулы для $R_{30}$ ). Очевидно, $\varphi$ и $D$ можно выбирать независимым образом, так как $\varphi$ зависит только от прослойки 12. тогда как $D$ зависит еще от $S_{13}$, а эта величина может быть сделана сколь угодно большой путем приближения $R_{23}$ к единице (см. формулу (21)). Наименьшее же возможное значение $S_{13}$ (см. формулу (9)) есть $\operatorname{sh} \varphi$, и как раз при этом значении $D$ получает свое наибольшее значение $e^{-\varphi}$. В этом предельном случае, когда несимметричность исчезает, пленка становится двусторонне просветленной, так как тогда $U_{13}=0$ и, согласно (12), также $U_{20}=0$ (см. [ $\left.\left.{ }^{3}\right]\right)$. Это согласуется с хорошо известным результатом (см $\left.\left[{ }^{4}\right]\right)$, гласящим, что у двусторонне просветленной поглощающей пленки коэффициент отражения равен с обеих сторон нулю.

Обращает на себя внимание то обстоятельство, отмеченное уже ранее ['], что даже при сколь угодно малом $\varphi$ можно выбрать $S_{13}$ столь большим (и, соответственно, $D$ малым), что $A_{03}$ будет близко к единице. Этот результат является неожнданным, так как малость $\varphi$ означает, что поглощающие слои в 12 очень тоңки. Тем не менее пленка поглощает євет (с одной стороны) почти полностью.

Отметим, наконец, что из двух вариантов условий несимметричности второй представляется несколько более удобным, так как в первом варианте выбор $S_{20}$ ограничивается условием $U_{20}<1$, тогда как во втором варианте подобного ограничения нет (вернее, это условие выполняется там автоматически). Однако, вопрос о целесообразности применения того или другого варианта зависит целиком от характера конкретной проблемы : 


\title{
Л ИТТ Р А Т Р А
}

1. П. К а рд, Теория многослойных несимметричных отражателей. Оптика и спектроскопия, $1961,10,384$.

2. П. К а р д, Новые рекуррентные формулы в теории многослойных оптических покрытий. Оптика и спектроскопия, $1960,9,95$.

3. П. К а р д, К теории поглощающих оптических покрытий. Изв. АН ЭССР. Сер. физ.матем. и техн. наук, 1960, 9, 250.

4. П. К а р д, Некоторые свойства поглощающих оптических покрытий. Изв. АН ЭССР. Сер. физ.-матем. и техн. наук, 1962, 11, 10.

Поступила в редакцию

13. III 1963

\section{MITTESOMMEETRILISTE PEEGLITE TEOORIAST}

\author{
P. Kard, \\ Eesti NSV Teaduste Akadeemia korrespondeeriv liige
}

Resümee

Mittesümmeetriliseks peegliks nimetatakse niisugust poolläbipaistvat kahekülgset peeglit, mille läbilaskvuskoefitsient on etteantud väärtusega ja mille peegeldumiskoefitsient ühel küljel võrdub nulliga ning teisel küljel on võimalikult suur. Vastavalt erinevad on ka neeldumiskoefitsiendid valguse langemisel peegli kummalegi küljele. Mittesümmeetrilise peegli võib valmistada mitmekihilise interferentssüsteemina, kusjuures selle kihtide hulgas peab olema vähemalt üks valgust neelav kiht. Juba varem [ $\left.{ }^{1}\right]$ olid tuletatud tarvilikud ning piisavad tingimused selleks, et niisugune interferentskile oleks mittesümmeetrilise peegli omadustega (valemid (10) ja (11)). Kujult erinevad, kuid sisuliselt ekvivalentsed mittesümmeetrilisuse tingimused vōib tuletada veel teisel viisil (valemid (15) ja 16)). Mölema variandi samaväärsust saab näidata otseste arvutuste teel, kuid samaväärsus järeldub ka sellest, et nii ühed kui teised tingimused on piisavad ning tarvilikud. Konkreetsete probleemide puhul on mōlema variandi tundmine oluliselt tähtis, sest olenevalt probleemi iseloomust võib kasulikumaks osutuda üks vōi teine.

Mittesümmeetrilise peegli peegeldumis- ja neeldumiskoefitsiendid võib lihtsal kujul avaldada (vt. valemid (26)) läbilaskvuskoefitsiendi $D$ ja kile neelavat osa iseloomustava suuruse $\varphi$ kaudu (vt. valem (8)). Joonisel 2 esitatakse need seosed graafiliselt. Suurused $D$ ja $\varphi$ varieeruvad teineteisest sōltumatult, kusjuures peegli mittesümmeetrilisus on seda suurem, mida väiksemad on $D$ ja $\varphi$.

Saabus toimetusse

13. III 1963

\section{ON THE THEORY OF ASYMMETRIC MIRRORS}

\author{
P. Kard, \\ Corresponding Member of the Academy of Sciences of the Estonian S.S.R.
}

\section{Summiary}

A double-sided semitransparent mirror is called asymmetric when its transmission coefficient has a prescribed value, while its reflection coefficient at the one side is zero and at the other is as large as possible. Accordingly, the absorption is at both sides unequal, too. An asymmetric mirror can be constructed in the shape of a multilayer interference film containing at least one absorbing layer. Already in an earlier paper [ ${ }^{1}$ ] the necessary and sufficient conditions are found ( $s$. formulas (10) and (11) in the present paper) for the film as an asymmetric mirror. In addition, another form of asymmetry conditions, though equivalent, can be deduced, too (s. formulas (15) and (16)). The equivalence of both versions can be shown by straightforward computations, and it is also manifest by the fact that the conditions in both versions are necessary and sufficient. The knowledge of both versions is of great importance in special problems, as either can be used to advantage according to the nature of the problem.

One can express the reflection and absorption coefficients of an asymmetric mirror in a simple manner (s. formula (26)) in terms of the transmission coefficient $D$ and of the characteristic quantity $\varphi(s$, formula $(8))$. The latter depends on the absorbing part of the film only. Figure 2 illustrates these expressions. Both the quantities $D$ and $\varphi$ can be independently varied. The smaller they are, the more asymmetric is the mirror.

Received

March 13th, 1963 